https://helda.helsinki.fi

\title{
The risk for end-stage renal disease is increased after burn
}

\section{Helanterä, llkka}

2016-03

Helanterä , I , Koljonen , V , Finne , P , Tukiainen , E \& Gissler , M 2016 , ' The risk for

end-stage renal disease is increased after burn ' , Burns , vol. 42 , no. 2 , pp. 316-321 . https://doi.org/10.1016/j.burn

http://hdl.handle.net/10138/223916

https://doi.org/10.1016/j.burns.2015.10.027

publishedVersion

Downloaded from Helda, University of Helsinki institutional repository.

This is an electronic reprint of the original article.

This reprint may differ from the original in pagination and typographic detail.

Please cite the original version. 


\title{
The risk for end-stage renal disease is increased after burn
}

\author{
Ilkka Helanterä ${ }^{a, 1}$, Virve Koljonen ${ }^{b, 1, *}$, Patrik Finne ${ }^{c, d}$, Erkki Tukiainen $^{b}$, \\ Mika Gissler ${ }^{e}$ \\ a Department of Transplant and Liver Surgery, University of Helsinki and Helsinki University Hospital, Helsinki, \\ Finland \\ ${ }^{\mathrm{b}}$ Department of Plastic Surgery, University of Helsinki and Helsinki University Hospital, Helsinki, Finland \\ ${ }^{\mathrm{c}}$ Department of Medicine, Division of Nephrology, University of Helsinki and Helsinki University Hospital, Helsinki, \\ Finland \\ ${ }^{\mathrm{d}}$ Finnish Registry for Kidney Diseases, Helsinki, Finland \\ ${ }^{\mathrm{e}}$ National Institute for Health and Welfare, Helsinki, Finland
}

\section{A R T I C L E I N F O}

Article history:

Accepted 26 October 2015

Keywords:

Acute kidney injury

End-stage renal disease

Complication

\begin{abstract}
A B S T R A C T
Objective: Acute kidney injury (AKI) commonly complicates burn. Recently, AKI has been suggested to be causally related to chronic end-stage renal disease (ESRD), but controversial data also exist. Our aim was to study the risk of ESRD after burn in a nationwide analysis. Methods: All burn patients undergoing hospitalization between 1998 and 2011 were identified from the National Hospital Discharge Register, and the data were linked with the Finnish Registry for Kidney Diseases, which includes all individuals receiving chronic renal replacement therapy (RRT) in Finland.

Results: Altogether 41,179 adults were treated at hospitals for burns in Finland between 1998 and 2011. Of these, 86 had a diagnosis of AKI related to the burn. Forty-three burn survivors had ESRD and RRT initiated related to or after the burn. The overall risk for ESRD after burn was increased (standardized incidence ratio, SIR, 2.40, 95\% CI 1.73-3.23) compared with the Finnish population. Standardized incidence ratio was 3.11 (95\% CI 1.66-5.32) in women and 1.89 (95\% CI 1.27-2.69) in men. Of these 43 patients, 38 had a specific non-burn-related diagnosis of ESRD identified in the registry, and ESRD was deemed unlikely to be directly related to the burn. In five patients, the diagnosis of ESRD was unknown cause of renal failure, and causality of the burn with ESRD was evaluated as plausible.

Conclusion: In conclusion, a significantly increased risk of ESRD was recorded after a severe burn. Our results do not support increased incidence of ESRD solely as a consequence of AKI due to burn, but burn may increase the risk of ESRD in patients with pre-existing chronic kidney disease.
\end{abstract}

(C) 2015 Elsevier Ltd and ISBI. All rights reserved.

\footnotetext{
* Corresponding author at: Department of Plastic Surgery, Töölö Hospital, P.O. Box 266, FI-00029 HUS, Finland. Tel.: +358 50427 1983; fax: +358947187217.

E-mail address: virve.koljonen@hus.fi (V. Koljonen).

1 These authors contributed equally to this work. http://dx.doi.org/10.1016/j.burns.2015.10.027 0305-4179/(C) 2015 Elsevier Ltd and ISBI. All rights reserved.
} 


\section{Introduction}

Acute kidney injury (AKI) is a frequent complication following severe burn [1-4]. The risk for AKI among burn patients increases with older age, larger percentage of total body surface burned, sepsis, multiple organ dysfunction and nephrotoxic antibiotics [5-8]. In burn patients, AKI carries an additional risk of morbidity and mortality [9-11]. Mortality rates ranging from $14 \%$ to $100 \%$ are reported $[3,4,9,12-14]$.

The pathophysiology of AKI in the setting of burn is most likely related to the longer duration of inflammatory response $[15,16]$ rather than to the decreased renal perfusion [17]. That said, intra-abdominal hypertension and abdominal compartment syndrome due to excessive fluid resuscitation $[18,19]$ or rhabdomyolysis $[20,21]$ may also lead to development of AKI [22,23].

Long-term dialysis after burn AKI is rare [24]. Traditionally, recovery from AKI has been expressed as recovery of kidney function with improvement of urine output and glomerular filtration rate, and AKI survivors are considered to have an excellent long-term prognosis [25]. However, recent research challenges this concept and non-burn AKI survivors seem to be at heightened risk for chronic kidney disease (CKD) and progressive renal failure $[26,27]$. The purpose of this epidemiological registry linkage study was to investigate the occurrence of end-stage renal disease (ESRD) among a large national cohort of burn patients obtained from the National Hospital Discharge Register.

\section{Materials and methods}

The Helsinki University Hospital Internal Review Board approved the study protocol. Permission to use the register information in scientific research was obtained from the National Institute of Health and Welfare (THL) after consulting the data protection authority. Specifically, permission to identify burn adult patients from the National Hospital Discharge Register and the Finnish Registry for Kidney Diseases was obtained from THL.

The Finnish Hospital Discharge Register, a national health care registry maintained by THL National Institute for Health and Welfare, has been maintained data since 1960. The registry includes all inpatient care since 1967 and outpatient care in public hospitals since 1998. The register collects information on hospitalizations (day of admission and discharge), outpatient visit (day of visit), diagnoses given during the inpatient care (ICD-10 codes since 1996) and surgical procedure codes (NOMESCO Classification of Surgical Procedures since 1997).

The Finnish Registry for Kidney Diseases, a national health care registry maintained by the Finnish Kidney and Liver Association, has been maintained data since 1964. The registry has an estimated coverage of $97-99 \%$ of all dialysis and kidney transplantation patients in Finland. The coverage of FRKD has been estimated several times during the existence of the registry. The latest estimate was performed in the annual report of 2010 [28]. All patients whose dialysis treatment lasts at least three months are recorded in the registry. Hospitals report to the registry information concerning kidney disease, including start date of renal replacement therapy (RRT), kidney disease diagnosis, modality of RRT - hemodialysis, peritoneal dialysis or kidney transplantation - laboratory variables and cardiovascular comorbidities. After start of RRT, data are updated annually. Written informed consent and permission to use the data anonymously in registry reports and for research purposes were obtained from all patients upon their entering the registry.

The register linkages in this study were done using the personal identity code (PIC) as a key. All citizens and permanent residents in Finland have a unique PIC, which was introduced in 1964-1967. The PIC code is given already in the birth hospital, and it remains unchanged during an individual's life span. The PIC is used in all main registers in Finland and allows reliable deterministic record linkage. The PICs were replaced by a study code after the linkage.

Inclusion criteria for this register linkage study comprised patient age $\geq 18$ years when the burn occurred, diagnosis with ICD-10 codes T20-T32.9 for burn and treatment in a hospital in Finland according to the National Hospital Discharge Register. The data of burn patients were reviewed to detect those who had been diagnosed with AKI before, during or after the burn accident with ICD-10 codes N17.0-N17.9 in 1.1.1998-31.12.2012. The cohort of burn patients was linked with the Finnish Registry for Kidney Diseases to identify all patients who were on chronic RRT before, during or after the burn. The causality between burn and ESRD was evaluated based on kidney disease diagnosis available in the Finnish Registry for Kidney Diseases and was recorded as either unlikely causality or plausible causality. The follow-up of the Finnish Registry for Kidney Diseases ended on 31.12.2013.

End-stage renal disease is defined as need for maintenance dialysis treatment for at least 3 months, or kidney transplantation. The incidence of ESRD is the number of patients with ESRD documented in the Finnish Register for Kidney Disease in relation to the total population of Finland at the same time period.

\subsection{Statistical analysis}

We calculated standardized incidence ratio (SIR) for the risk of ESRD after burn by using national rates from the Finnish Registry for Kidney Diseases during the study period. We performed survival analysis by generating a Kaplan-Meier survival plot until first event of end-stage renal disease. The right-censoring occurred only if the patient was alive without event occurrence at last follow-up in 31 December 2011.

\section{Results}

\subsection{All patients}

During the study period 41,179 adults in Finland were treated for burns at hospitals as in- or outpatients. The total number of follow-up years was 374,603, with a mean of 9.1 years per patient. Total treatment periods numbered 115,828 . Of the patients, $48 \%$ had only one treatment period; one treatment period refers to one hospital admission, including the 


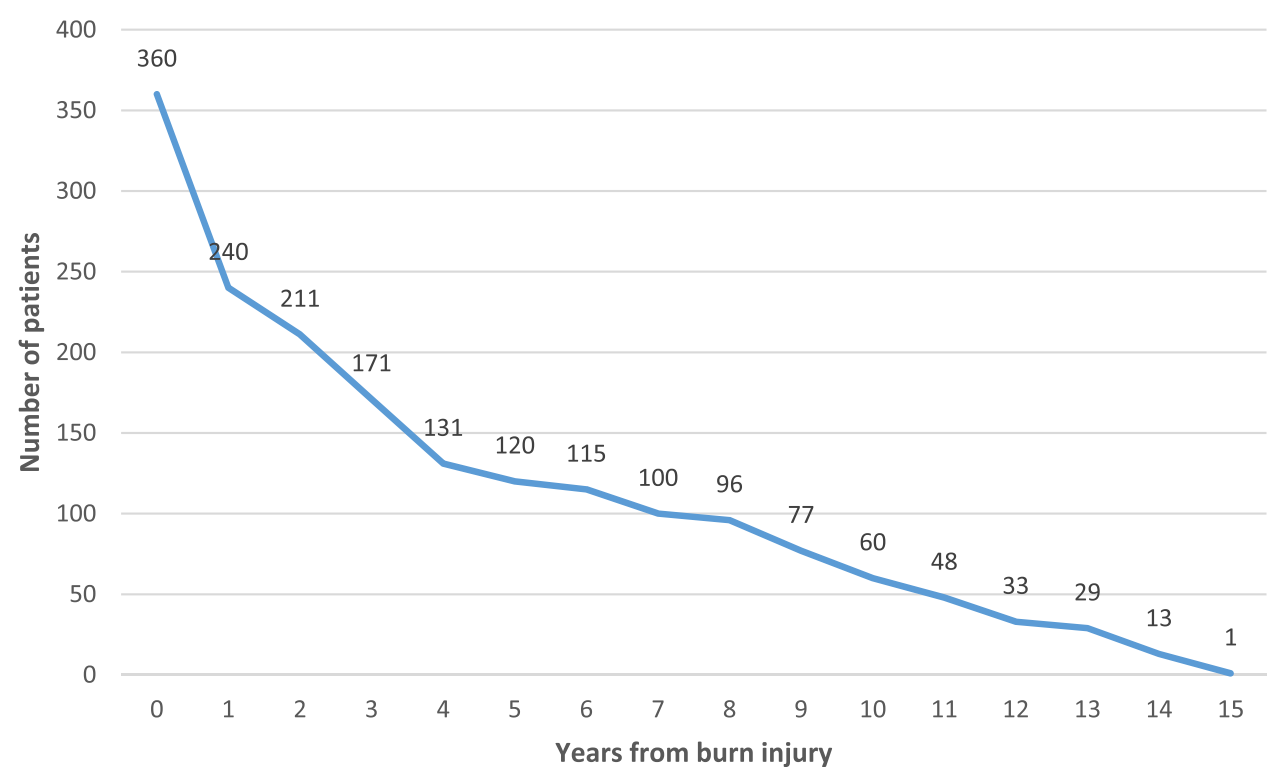

Fig. 1 - Number of patients diagnosed with acute kidney injury after burn in Finland in 1998-2012.

emergency presentation admission associated with the same hospital admission. Of the 41,255 burn patients, 37,323 (90.5\%) were not diagnosed with kidney disease in either of the registries before, during or after the burn.

\subsection{Acute renal failure}

Fig. 1 illustrates the occurrence of AKI during the post-burn follow-up period. Altogether, 1660 burn survivors were diagnosed with AKI. Fig. 2 illustrates the incidence density of AKI after the burn. During the first year post-injury we recorded 345 AKI diagnosis, which is $20.8 \%$ of all AKI diagnoses, yielding an incidence of 1059 per 100,000 followup years. In 86 patients, AKI was diagnosed simultaneously with the burn. During the whole follow-up the incidence of AKI after burn was 510 per 100,000 person-years.

\subsection{End-stage renal disease}

We recorded 43 burn survivors in the Finnish Registry for Kidney Diseases who had ESRD and RRT initiated simultaneously with or after the burn. The SIR for ESRD after burn for all patients was 2.40 (95\% CI 1.73-3.23) compared with the general Finnish population. The risk for ESRD was increased in both genders, but more notably in women (SIR 3.11, 95\% CI 1.66-5.32) than in men who were burn survivors (SIR 1.89, 95\% CI 1.27-2.69) (Table 1).

\subsection{Association of end-stage renal disease with burn}

Table 2 illustrates in detail the characteristics of the patients who were diagnosed with ESRD after burn. Shortly, most were male and hemodialysis was started first in the majority of the patients. After the initial dialysis period, eight received a kidney transplantation. Of the 43 patients who developed ESRD after the burn, altogether 26 died; 16 due to cardiovascular causes, 5 due to infectious causes, and 5 due to other causes, of which none were burn-related.

Of the 43 patients who started RRT after the burn, 38 had a specific diagnosis of ESRD, including diabetic nephropathy $(\mathrm{N}=27)$, autosomal dominant polycystic kidney disease $(N=3)$, IgA nephropathy $(N=3)$, Alport syndrome $(N=1)$, secondary amyloidosis $(N=1)$, multiple myeloma $(N=1)$, nephrosclerosis $(N=1)$ and focal and segmental glomerulosclerosis $(N=1)$, recorded in the registry. Thus, a causal association between burn AKI and ESRD was deemed unlikely, and burn was not considered a direct cause of ESRD.

Among these 38 patients, RRT was initiated within one year from the burn in three patients (at 1, 2 and 9 months after burn hospitalization, respectively), whereas in the other 35 patients RRT was started at a median of 5.2 years (range 1.3-14.8 years) after burn.

In the remaining five patients, the diagnosis of ESRD was unknown cause of renal failure (ICD-10 code N18.9). In two of these patients, maintenance RRT was started within one year from the burn hospitalization (one week and 12 months after burn hospitalization, respectively), whereas in the other three patients with an unknown cause of renal failure, RRT was initiated at 5.3, 8.7 and 9.7 years after burn, respectively. A causal association between burn and ESRD was considered plausible.

\section{Discussion}

The risk for ESRD after burn in our patient pool was increased significantly. The risk rose by nearly 2.5 -fold compared with the general population. However, given that the majority of patients with ESRD after burn were diagnosed with a specific kidney disease as the cause of ESRD, we deemed it unlikely that burn directly caused ESRD. Instead, we speculate that burn contributed to accelerated deterioration of kidney 

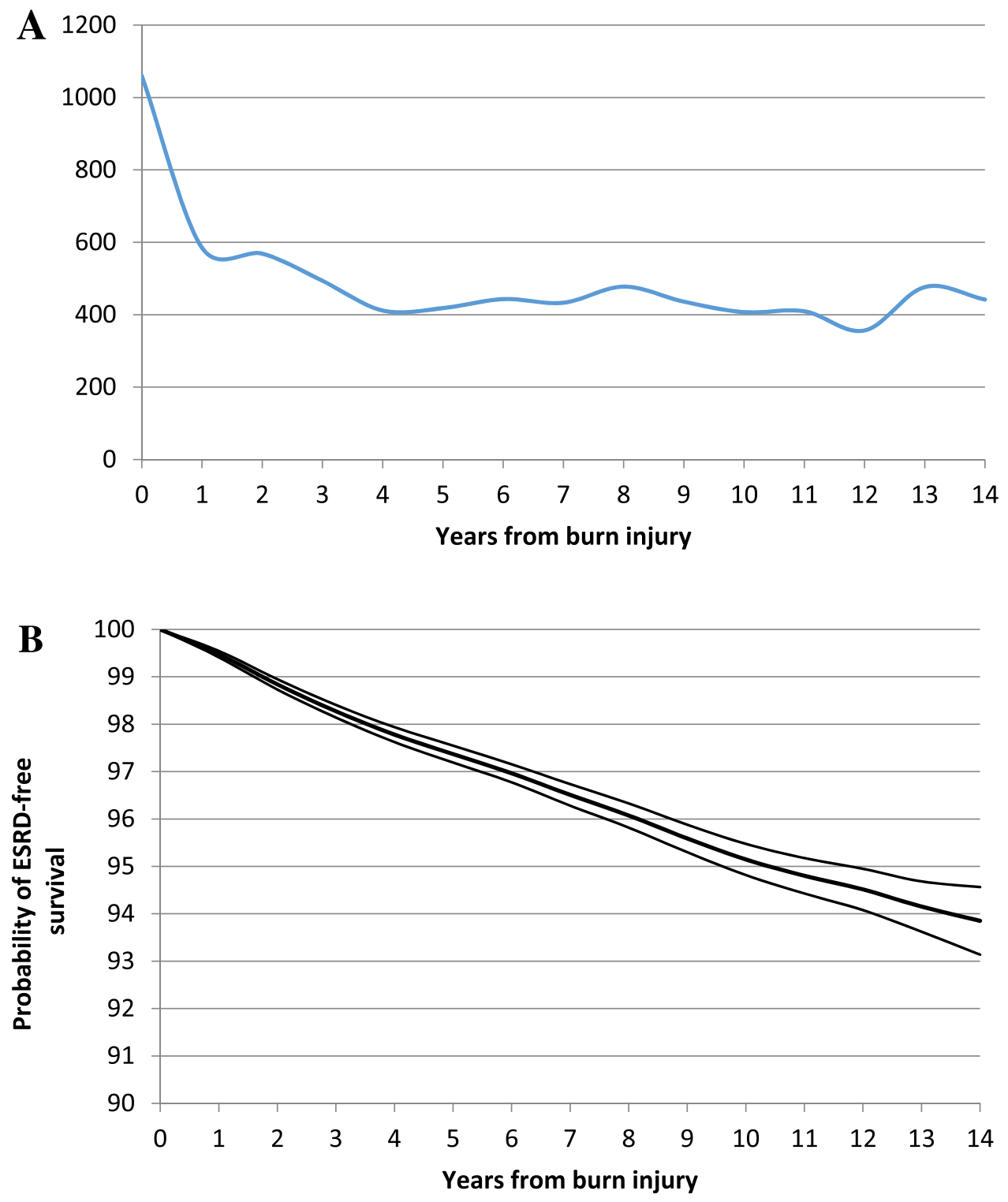

Fig. 2 - (A) Number of patients diagnosed with acute kidney injury after burn per 100,000 person years. (B) Kaplan-Meier survival curve with $95 \%$ confidence intervals for burn injuries.

function in patients with underlying CKD, explaining the increased incidence. Traditionally, patients with complete recovery from AKI have been suggested to have an excellent prognosis, but recent data have challenged this concept

Table 1 - Incidence of end-stage renal disease after burn compared with incidence in the whole Finnish population. $\mathrm{O} / \mathrm{E}=$ observed/expected.

\begin{tabular}{llll} 
& \multicolumn{1}{c}{ Male } & Female & \multicolumn{1}{c}{ All } \\
\hline $\mathrm{N}$ & 27,465 & 13,714 & 41,179 \\
Observed & 30 & 13 & 43 \\
Expected & 15.90 & 4.18 & 17.95 \\
O/E = standardized & 1.89 & 3.11 & 2.40 \\
$\quad$ incidence ratio & & & \\
95\% CI & $1.27-2.69$ & $1.66-5.32$ & $1.73-3.23$ \\
Per 100,000 person years & 16.8 & 16.3 & 16.6 \\
\hline
\end{tabular}

$[29,30]$. Moreover, controversy exists as to whether AKI is causally related to chronic progressive renal failure or whether several residual confounding factors, such as baseline kidney function, shared risk factors or ascertainment bias (sicker patients have more follow-up assessments), might explain the association [31].

In this study, the vast majority of the ESRD patients had a specific diagnosis of kidney disease, and this association may be based on the residual confounding factors mentioned above. In the non-burn literature on critically ill patients with AKI, the succeeding risk for CKD and ESRD is increased with or without RRT [31-33]. In our study, we were unable to determine whether the burn accelerated the deterioration of kidney function in patients with pre-existing renal disease, suggesting an acute-on-chronic type of injury trait. Even mild chronic impairment of kidney function significantly increases the risk of AKI [34]. Acute-on-chronic type of kidney injury 
Table 2 - Characteristics of patients diagnosed with endstage renal disease after burn.

\begin{tabular}{ll} 
& \multicolumn{1}{c}{$\begin{array}{c}\text { Patients } \\
\text { diagnosed } \\
\text { with ESRD } \\
\text { after burn } \\
(\mathrm{N}=43)\end{array}$} \\
\hline Mean age at burn, years (range) & $52(10-81)$ \\
Mean age at ESRD diagnosis, years (range) & $57(17-81)$ \\
Male/female (\%) & $30 / 13(70 / 30)$ \\
Mortality during follow-up (\%) & $26(60)$ \\
Mean length of follow-up, years (range) & $8.6(1.5-14.4)$ \\
Mean time from burn to death, years (range) & $8.1(1.5-14.1)$ \\
Hemodialysis as first treatment modality (\%) & $28(65)$ \\
[vs. peritoneal dialysis] & \\
Patients receiving a kidney transplant & $8(19)$ \\
during follow-up (\%) & \\
Patients with history of comorbid & \\
condition, (\%) & \\
Angina pectoris & $8(19)$ \\
Myocardial infarction & $7(16)$ \\
Peripheral arteriosclerosis with & $7(16)$ \\
amputation or other surgery & \\
Stroke & $7(16)$ \\
Left ventricular hypertrophy & $6(14)$ \\
Cardiac decompensation & $5(12)$ \\
Coronary bypass surgery & $3(7)$ \\
\hline ESRD, end-stage renal disease. & \\
\hline
\end{tabular}

during hospitalization results in poorer survival [35], and the severity of the AKI correlates directly with the progression to CKD [36].

Previous literature shows that the risk for AKI among patients with major burns can be as high as $24 \%$ [3]. In this study, we established an incidence rate of 17 per 100,000 person-years. The National Hospital Discharge register automatically collects data on all hospitalizations occurring in Finland. However, the reliability of the registry data depends on the quality of the reporting of individual hospitals. Definitions for AKI have undergone major changes since the early years of this analysis (starting from 1998). Similarly, additional diagnoses during hospitalization, such as AKI, probably have not been reported accurately in the registry, especially in the early years of the study. Therefore, AKI diagnoses associated with burns are most likely underreported in the National Hospital Discharge Register, and the occurrence of AKI in association with burns in our study cannot be established precisely.

To our knowledge, none of the previous studies addressing the incidence of ESRD following AKI in burn patients are based on a nationwide database. A main strength of our study is the use of two nationwide registries that allow reliable assessment of the incidence of ESRD among a large population of burn patients. Other strengths include the high completeness and accuracy of information available from the Finnish Registry for Kidney Diseases [37] and the long time-window of the follow-up. A further strength is the large burn patient cohort with register data linkage for understanding the evolution of ESRD in patients with burns. We thoroughly consider several potential biases in the registry data and in our analyses. Inherent limitations are that we did not have individual-level data on the severity of burn and patient-related risk factors since only data included in the registries were available for our analyses. According to local rules and the permission to obtain data from the registries, the personal identity codes were encrypted after the record linkage, and no further linkages or review of individual-level patient files were unfortunately possible. In addition, the data linkage between registries allows us to only study associations, drawing no conclusions on true causality. However, with the very large number of burn patients in our analysis and the almost complete coverage of ESRD patients in Finland of the Finnish Registry for Kidney Diseases, it is unlikely that we have missed patients with ESRD occurring after burn.

To conclude, we show a statistically increased risk of ESRD after severe burn. However, our results do not support the notion that ESRD after burn is directly associated with burn AKI. Based on our data, burns are not directly causally associated with ESRD, but may increase the risk of ESRD in patients with pre-existing CKD.

\section{R E F E R E N C E S}

[1] Brusselaers N, Monstrey S, Colpaert K, Decruyenaere J, Blot SI, Hoste EA. Outcome of acute kidney injury in severe burns: a systematic review and meta-analysis. Intensive Care Med 2010;36:915-25.

[2] Mosier MJ, Pham TN, Klein MB, Gibran NS, Arnoldo BD, Gamelli RL, et al. Early acute kidney injury predicts progressive renal dysfunction and higher mortality in severely burned adults. J Burn Care Res 2010;31:83-92.

[3] Steinvall I, Bak Z, Sjoberg F. Acute kidney injury is common, parallels organ dysfunction or failure, and carries appreciable mortality in patients with major burns: a prospective exploratory cohort study. Crit Care 2008;12:R124

[4] Palmieri T, Lavrentieva A, Greenhalgh DG. Acute kidney injury in critically ill burn patients. Risk factors, progression and impact on mortality. Burns 2010;36: 205-11.

[5] Hu JY, Meng XC, Han J, Xiang F, Fang YD, Wu J, et al. Relation between proteinuria and acute kidney injury in patients with severe burns. Crit Care 2012;16:R172.

[6] Yang HT, Yim H, Cho YS, Kym D, Hur J, Kim JH, et al. Assessment of biochemical markers in the early post-burn period for predicting acute kidney injury and mortality in patients with major burn injury: comparison of serum creatinine, serum cystatin-C, plasma and urine neutrophil gelatinase-associated lipocalin. Crit Care 2014;18:R151.

[7] Holm C, Horbrand F, von Donnersmarck GH, Muhlbauer W. Acute renal failure in severely burned patients. Burns 1999;25:171-8

[8] Stewart IJ, Tilley MA, Cotant CL, Aden JK, Gisler C, Kwan $\mathrm{HK}$, et al. Association of AKI with adverse outcomes in burned military casualties. Clin J Am Soc Nephrol 2012;7:199-206.

[9] Coca SG, Bauling P, Schifftner T, Howard CS, Teitelbaum I, Parikh CR. Contribution of acute kidney injury toward morbidity and mortality in burns: a contemporary analysis. Am J Kidney Dis 2007;49:517-23.

[10] Chung KK, Stewart IJ, Gisler C, Simmons JW, Aden JK, Tilley MA, et al. The Acute Kidney Injury Network (AKIN) criteria applied in burns. J Burn Care Res 2012;33:483-90. 
[11] Lopes JA, Jorge S, Neves FC, Caneira M, da Costa AG, Ferreira AC, et al. An assessment of the RIFLE criteria for acute renal failure in severely burned patients. Nephrol Dial Transplant 2007;22:285.

[12] Kim GH, Oh KH, Yoon JW, Koo JW, Kim HJ, Chae DW, et al. Impact of burn size and initial serum albumin level on acute renal failure occurring in major burn. Am J Nephrol 2003;23:55-60.

[13] Mustonen KM, Vuola J. Acute renal failure in intensive care burn patients (ARF in burn patients). J Burn Care Res 2008;29:227-37.

[14] Chrysopoulo MT, Jeschke MG, Dziewulski P, Barrow RE, Herndon DN. Acute renal dysfunction in severely burned adults. J Trauma 1999;46:141-4.

[15] Jeschke MG, Mlcak RP, Finnerty CC, Norbury WB, Gauglitz GG, Kulp GA, et al. Burn size determines the inflammatory and hypermetabolic response. Crit Care 2007;11:R90.

[16] Wan L, Bellomo R, Di Giantomasso D, Ronco C. The pathogenesis of septic acute renal failure. Curr Opin Crit Care 2003;9:496-502.

[17] Colpaert K, Hoste EA. Acute kidney injury in burns: a story of volume and inflammation. Crit Care 2008;12:192.

[18] Strang SG, Van Lieshout EM, Breederveld RS, Van Waes OJ. A systematic review on intra-abdominal pressure in severely burned patients. Burns 2014;40:9-16.

[19] Markell KW, Renz EM, White CE, Albrecht ME, Blackbourne LH, Park MS, et al. Abdominal complications after severe burns. J Am Coll Surg 2009;208:940-7 [discussion 7-9].

[20] Lazarus D, Hudson DA. Fatal rhabdomyolysis in a flame burn patient. Burns 1997;23:446-50.

[21] Koljonen V. Hot air sauna burns - review of their etiology and treatment. J Burn Care Res 2009;30:705-10.

[22] Sandhu G, Mankal P, Gupta I, Ranade A, Bansal A, Jones J. Pathophysiology and management of acute kidney injury in the setting of abdominal compartment syndrome. Am J Ther 2014;21:211-6.

[23] Sugrue M, Jones F, Deane SA, Bishop G, Bauman A, Hillman $\mathrm{K}$. Intra-abdominal hypertension is an independent cause of postoperative renal impairment. Arch Surg 1999;134:1082-5.

[24] Soltani A, Karsidag S, Garner W. A ten-year experience with hemodialysis in burn patients at Los Angeles County + USC Medical Center. J Burn Care Res 2009;30:832-5.
[25] Leung KC, Tonelli M, James MT. Chronic kidney disease following acute kidney injury-risk and outcomes. Nat Rev Nephrol 2013;9:77-85.

[26] Lo LJ, Go AS, Chertow GM, McCulloch CE, Fan D, Ordonez JD, et al. Dialysis-requiring acute renal failure increases the risk of progressive chronic kidney disease. Kidney Int 2009;76:893-9.

[27] Harel Z, Bell CM, Dixon SN, McArthur E, James MT, Garg AX, et al. Predictors of progression to chronic dialysis in survivors of severe acute kidney injury: a competing risk study. BMC Nephrol 2014;15:114.

[28] Finnish Registry for Kidney Diseases. Finnish Registry for Kidney Diseases. Report 2010; 2010, http://www.musili.fi/ files/456/Report_2010.pdf [18.09.15].

[29] Jones J, Holmen J, De Graauw J, Jovanovich A, Thornton S, Chonchol M. Association of complete recovery from acute kidney injury with incident CKD stage 3 and all-cause mortality. Am J Kidney Dis 2012;60:402-8.

[30] Chawla LS, Kimmel PL. Acute kidney injury and chronic kidney disease: an integrated clinical syndrome. Kidney Int 2012;82:516-24.

[31] Rifkin DE, Coca SG, Kalantar-Zadeh K. Does AKI truly lead to CKD? J Am Soc Nephrol 2012;23:979-84.

[32] Wald R, Quinn RR, Adhikari NK, Burns KE, Friedrich JO, Garg AX, et al. Risk of chronic dialysis and death following acute kidney injury. Am J Med 2012;125:585-93.

[33] Coca SG, Singanamala S, Parikh CR. Chronic kidney disease after acute kidney injury: a systematic review and metaanalysis. Kidney Int 2012;81:442-8.

[34] Hsu CY, Ordonez JD, Chertow GM, Fan D, McCulloch CE, Go AS. The risk of acute renal failure in patients with chronic kidney disease. Kidney Int 2008;74:101-7.

[35] Wu V-C, Huang T-M, Lai C-F, Shiao C-C, Lin Y-F, Chu T-S, et al. Acute-on-chronic kidney injury at hospital discharge is associated with long-term dialysis and mortality. Kidney Int 2011;80:1222-30.

[36] Chawla LS, Amdur RL, Amodeo S, Kimmel PL, Palant CE. The severity of acute kidney injury predicts progression to chronic kidney disease. Kidney Int 2011;79:1361-9.

[37] Stengel B, Billon S, Van Dijk PC, Jager KJ, Dekker FW, Simpson K, et al. Trends in the incidence of renal replacement therapy for end-stage renal disease in Europe, 1990-1999. Nephrol Dial Transplant 2003;18:1824-33. 